\title{
Compact Optical Comb Generator using InP Tunable Laser and Push-pull Modulator
}

\author{
R. Slavík, S.G. Farwell, M.J. Wale, and D.J. Richardson
}

\begin{abstract}
We have investigated the suitability of InP-based push-pull modulators to generate flat optical comb spectra. The residual amplitude modulation that was a consequence of driving the modulator well above its design voltage (up to $4 V_{\pi}$ ) is demonstrated to have no adverse effect for the comb generation. We also demonstrate generation of a comb with spacing equal to twice the repetition rate via control of the relative phase of the RF signals.
\end{abstract}

Index Terms-Optical frequency comb, Mach-Zehnder modulator, semiconductor optical modulator.

\section{INTRODUCTION}

Optical frequency combs generated in the telecom C-band have attracted a lot of attention in the literature over the past decade [1-15]. The most popular generation technique is based on modulating an input CW laser beam within a cascade of modulators that operate on the signal amplitude, phase, or polarization (or combinations thereof). This technique has several key advantages: it can be realized using off-the shelf components available in most photonics laboratories, allows a tunable comb tone spacing, the number of frequency tones can be scaled up by using more modulators, the phase noise properties are given by the seed laser only (which can be narrow-linewidth), and it can produce a flat comb spectrum through an appropriate choice of modulation parameters. Other approaches presented in the literature use various configurations of lasers [1,2], gainswitched semiconductor lasers [3], and optical feedback loops $[4,5]$. Laser-based approaches generally produce combs lacking tunability of the comb spacing and the linewidth of each comb tone is modest (typically $10 \mathrm{MHz}$ [1]). Feedback loops can be active [4,6], or passive [7], resulting in limited frequency tunability and/or spectral flatness. Gain-switched semiconductor lasers are simple and efficient, but produce combs inherently limited by the bandwidth of the laser (e.g., about 10 tones at $10 \mathrm{GHz}$ [3]).

Among the techniques using optical modulators, there is one that stands out, as it needs only a single modulator to produce flat frequency combs [8]. The modulator is of the Mach-Zehnder configuration with electrodes in both its arms ('dual-electrode' modulator). Such a modulator is commercially-available, as it is used for a push-pull operation in optical communications (reducing the necessary RF voltage

Manuscript received XXX. This research has been supported by EPSRC Fellowship grant agreement no. EP/K003038/1. R. Slavík and D.J. Richardson are with the Optoelectronics Research Centre, University of Southampton, Southampton SO17 1BJ, U.K. (e-mail: r.slavik@soton.ac.uk). S.G. Farwell and M.J. Wale are with Oclaro, Caswell, Towcester, Northamptonshire, NN12 8EQ, U.K. by a factor of two for amplitude-modulated signals), or for generation of binary phase shift keyed signals. This combgeneration configuration was first investigated theoretically [8] and it was concluded that a flat comb response can always be obtained if the following condition is met:

$$
\Delta A \pm \Delta \phi=\frac{\pi}{2},
$$

where $\Delta \phi$ is the phase difference between the two RF drive signals (see Fig. 1) and $\Delta A=\left(A_{1}-A_{2}\right) / 2$ (see Fig. 1 for $\left.A_{1,2}\right)$ is given by the difference in drive voltages applied to the two RF electrodes:

$$
A_{1,2}=\frac{\pi}{2 V_{\pi}} V_{p-p}
$$

where $V_{p-p}$ is the applied peak-to-peak voltage and $V_{\pi}$ is the electrode voltage for $\pi$ phase-shift within each individual arm. Shortly after this theoretical analysis, comb generation was confirmed experimentally using a $\mathrm{LiNbO}_{3}$ modulator [9], generating 19 x $10 \mathrm{GHz}$-spaced comb tones with an amplitude ripple of $4.3 \mathrm{~dB}$. The comb conversion efficiency (total comb power at the output versus the CW light power at the input) was $-20 \mathrm{~dB}$ and the RF powers sent to the two electrodes were 35.9 and $36.4 \mathrm{dBm}$, respectively. Tunability over $50 \mathrm{~nm}$ was demonstrated using a tunable input laser. This simple optical configuration obviously attracted further attention and work has been done to decrease the drive voltage via optimization of the RF signals [10] and by employing alternative materials for the modulator, such as InP [11,12], or silicon-organic hybrid (SOH) [13]. Unfortunately, only a modest number of tones was achieved in these systems - generally less then 10 , although some of them were at high comb spacing (e.g., up to 7 tones at $40 \mathrm{GHz}$ spacing in the SOH device [13]). This smaller number of comb tones may already be sufficient for some applications, e.g., Ortohogonal Frequency Domain Multiplexing (OFDM) [13], but a higher number of comb tones would be desirable to allow for a larger number of applications or higher-capacity (e.g., > 1Tbit/s OFDM generation).

It is perhaps worth mentioning that another 'singlemodulator' configuration has been investigated, based on a double-nested Mach-Zehnder modulator used in today's coherent communications for 'In-phase and Quadrature' (IQ) modulation. It was first suggested in [14], where 9 comb tones were generated using two RF signals (i.e., the same number of drive voltages as in the dual-electrode Mach-Zehnder modulator), or using just a single-drive RF signal [15], where 
it was shown that a maximum of 7 tones with equal power can be generated.

Here, we report on a significant improvement in combgeneration based on the InP platform that may make this technology appealing for practical applications. First, our device has the (C-band-tunable) seed laser integrated with the dual-electrode Mach-Zehnder modulator in a single package (TL7000 from Oclaro) and which can also be realized in a fully monolithic form (as is done in the TL8800 transceiver). Further, we investigate experimentally the maximum number of comb tones that can be generated given the specific InP modulator performance, obtaining up to 29 comb tones within a 3-dB bandwidth - about three times the number previously reported using an InP modulator. We also demonstrate comb generation at twice the RF signal frequency (20 GHz spacing using 10-GHz RF signals) with over $25 \mathrm{~dB}$ suppression of the fundamental frequency $(10 \mathrm{GHz})$ signal.

\section{PRINCIPLE AND SET-UP}

Our set-up is shown in Fig. 1. The tunable laser emitting $13 \mathrm{dBm}$ is co-packaged with a push-pull modulator (total loss including input/output coupling loss of about $6 \mathrm{~dB}$ ) with a bandwidth of $\sim 10 \mathrm{GHz}$. Fuller details are given in $[16,17]$.

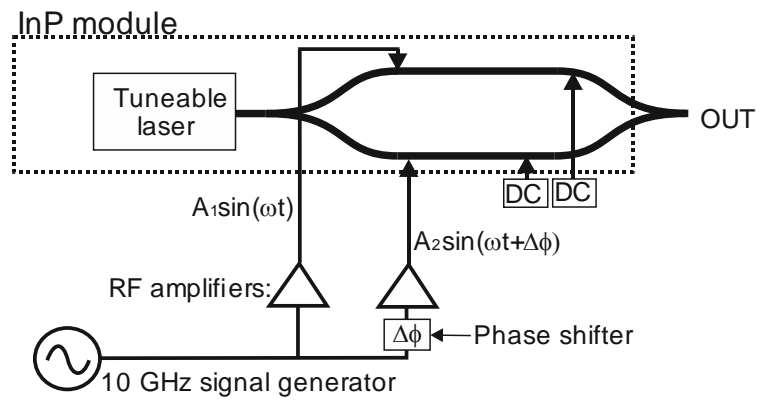

Fig. 1: Set-up.

Besides Eq. (1), Sakamoto et al [8] calculated that the number of tones obtainable in an ideal dual-electrode MachZehnder modulator is $\sim \pi A$, where $A=\left(A_{1}+A_{2}\right) / 2$. Considering Eq. (2) and $A_{1} \cong A_{2}$, the number of comb tones $N$ is given by:

$$
N \sim \frac{\pi^{2}}{2 V_{\pi}} V_{p-p} .
$$

It is worth mentioning that for an InP modulator, we expect to get a slightly different result. In a semiconductor material, the refractive index change is obtained through the KramersKronig relation due to sharp bandgap absorption. The bandgap is set such that the seed CW light to be modulated is on the long-wavelength side of the bandgap (thus experiencing minimum loss). By applying voltage, the bandgap absorption edge wavelength shifts towards the seed laser wavelength, changing the refractive index experienced by the seed laser light (due to the strong disperison close to the bandgap edge). When the seed CW laser wavelength is set too close to the bandgap, the refractive index change can be large, but it is accompanied by a large change in the absorption. In optimising a telecommunications modulator one seeks to minimise the residual amplitude modulation by operating far from the bandgap edge, but this comes at the expense of a higher $\mathrm{V}_{\pi}$. In this design regime we expect the InP modulator peformance for optical comb generation to be similar to that of $\mathrm{LiNbO}_{3}$ modulators. However for either technology, wide comb generation requires much higher RF drive powers, requiring the telecom-optimized modulator to be driven harder and thus inevitably getting closer to the bandgap edge. Fortunately, this is expected to reduce $\mathrm{V}_{\pi}$ (and thus increase the number of generated comb tones), but introduces the aforementioned additional amplitude modulation. Thus, we expect to have a mixture of amplitude and phase modulation at each modulator electrode, which is known to produce an asymmetric spectral profile.

We measured $V_{\pi}$ of our modulator to be $2.7 \mathrm{~V}$ (for small bias voltages). To study the influence of the ratio of RF powers sent to the two electrodes, we generated RF signals with different strengths by inserting an RF attenuator in front of one of the RF amplifiers. In the following experiments, we set the RF power ratio in the two arms to $6 \mathrm{~dB}$ and $1 \mathrm{~dB}$, respectively.

\section{EXPERIMENTAL RESULTS AND DISCUSSION}

When keeping the RF power ratio at $6 \mathrm{~dB}$, we slowly increased the RF power and tried to tune the two DC bias voltages and the phase difference $\Delta \phi$ to obtain flat spectra with as large a 3-dB bandwidth as possible. As we increased the RF power, we generated more and more comb tones, obtaining 20 tones within a 3-dB bandwidth at RF powers of $27 / 21 \mathrm{dBm}$. No further broadening was obtained at higher RF levels. We attribute this to an increase in the amplitude modulation, as we approach the bandgap absorption edge. Since approaching the bandgap edge is also associated with increased transmission loss we did not increase the RF power any further. The result for $27 / 21 \mathrm{dBm}$ of RF power is shown in Fig. 2a. Fig. 2b shows spectra when we turned off one or other RF signal respectively. From theory, we would expect a Bessel spectral profile for the comb in Fig. 2b [8], as was previously observed with an $\mathrm{LiNbO}_{3}$ device [9]. Although there are some spectral features similar to those observed in [9] (e.g. a central tone about $10 \mathrm{~dB}$ above the others), the spectrum is slightly asymmetric, suggesting we do not have the pure phase modulation assumed in theory [8]. We believe this is due to the 'parasitic' amplitude modulation present at large driving voltages, as discussed previously. Fortunately, as shown in Fig. 2b, this asymmetry can be set to be opposite for the two RF electrodes, thereby compensating each-other and producing a relatively symmetric frequency comb, as shown in Fig. 2a. The output power within a 3-dB comb bandwidth (20 tones) was measured to be over $0.6 \mathrm{~mW}$.

In the next experiment, we used an RF power difference of only $1 \mathrm{~dB}$. As in the previous experiment, we kept increasing the RF power and optimized the biases and phase shift to achieve a flat comb spectrum. Fig. 3 illustrates a trend that was quite general. For a given RF power, we could obtain an essentially flat comb spectrum, as shown in Fig. 3 (bottom curve), by optimizing the DC bias voltages and the phase $\Delta \phi$. It was possible to get more comb tones by allowing a dip in the middle of the spectrum, Fig. 3, middle and upper curve. 
We also see that a wider comb with a more pronounced dip is generated with lower conversion efficiency.

As we kept increasing the RF power, we continued to get more and more comb tones up to the $26 / 27 \mathrm{dBm}$ level, as shown in Fig. 3. Beyond this point no additional tones were generated and higher attenuation was observed. Fig. 4 compares the comb generated at RF powers of $26 / 27 \mathrm{dBm}$ and 25/26 dBm. In both cases, we tried to generate a comparable number of tones. Whilst with $26 / 27 \mathrm{dBm}$ the comb is very flat (Fig. 4a), when the lower RF power is used, the same number of comb tones could only be achieved at the expense of a dip in the middle of the spectrum (Fig. 4b). However, the conversion efficiency is comparable in both cases (power-percomb tone), as seen in Fig. 4. Thus, when targeting a flat comb, a higher RF power is preferred, as the comb has better uniformity and - at the same time - the conversion efficiency is not compromised. Slightly better results in terms of conversion efficiency were obtained earlier, Fig. 2, where a larger difference in RF powers was used (27/21 dBm), albeit at a slightly lower number of comb tones (20 vs. 22). This shows that the comb generator is relatively insensitive to the difference in the RF driving powers.

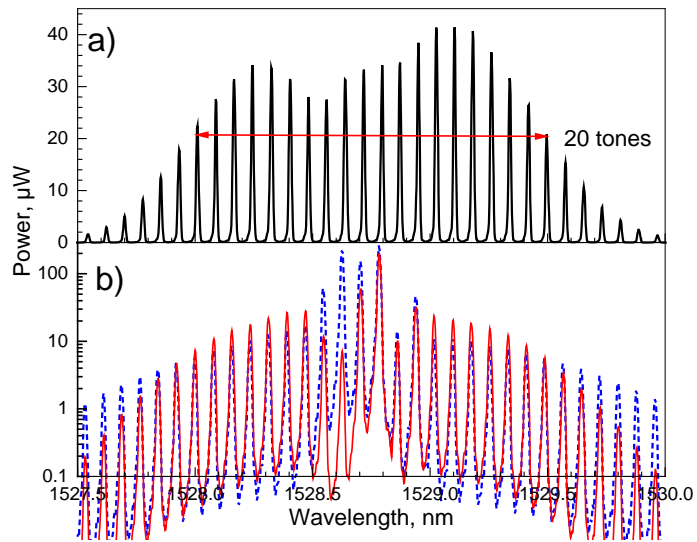

Fig. 2: Comb response when both RF signals are on (a) and when one of them is off (b) without changing the bias voltages. The electrical RF power was 27 and $21 \mathrm{dBm}$, respectively.

Based on the theory calculated for an ideal dual-electrode modulator, Eq. (3), $V_{\pi}$ of our modulator of $2.7 \mathrm{~V}$, and for the average $V_{p-p}$ applied to the two RF electrodes of $9.4 \mathrm{~V}$ (power of $26.5 \mathrm{dBm}$ ), we would expect the generation of 17 comb tones. However, we obtained 29 tones, suggesting our $V_{\pi}$ was - as we have expected - reduced as we biased the modulator higher to allow for deeper modulation, as discussed earlier. It is perhaps worth comparing our result (29 tones, ripple $<3$ $\mathrm{dB}$, RF powers of $27 \mathrm{dBm}$ ) with that obtained with a $\mathrm{LiNbO}_{3}$ (19 tones, ripple $>4 \mathrm{~dB}$, RF powers of $36 \mathrm{dBm}$ ) [9]), clearly showing the advantages of the InP platform.

In all the above results, we always optimized the RF phase shift $\Delta \phi$. It is interesting to see what happens when this phase is tuned. We found that as it is tuned away from its optimum value, a quasi-periodic spectral variation is induced (an example is shown in Fig. 5). When tuning the phase $\Delta \phi$ from its optimum by $\pi$, this spectral variation reaches $20 \mathrm{GHz}$. This implies that every other comb tone could be suppressed, effectively generating a $20-\mathrm{GHz}$ comb. Fig. 6a shows the extent to which alternate tones can be suppressed. Measuring across eleven 20-GHz spaced comb tones (occupying a bandwidth of $1.6 \mathrm{~nm}$ ), the suppression of the residual $10-\mathrm{GHz}$ tones is better than $25 \mathrm{~dB}$. By fine-tuning of the phase $\Delta \phi$, odd or even $10-\mathrm{GHz}$ comb tones can be suppressed, as shown in Fig. 6b. Here, we can also appreciate that the shape of the comb is not perfectly flat, clearly demonstrating that the two DC biases do not in themselves provide enough control to generate a flat comb and that control of the phase $\Delta \phi$ is also needed. Eq. (1) suggests that a flat comb response could be achieved if $\Delta A$ could have been controlled instead of $\Delta \phi$. Unfortunately, we did not have a suitable attenuator to confirm this.

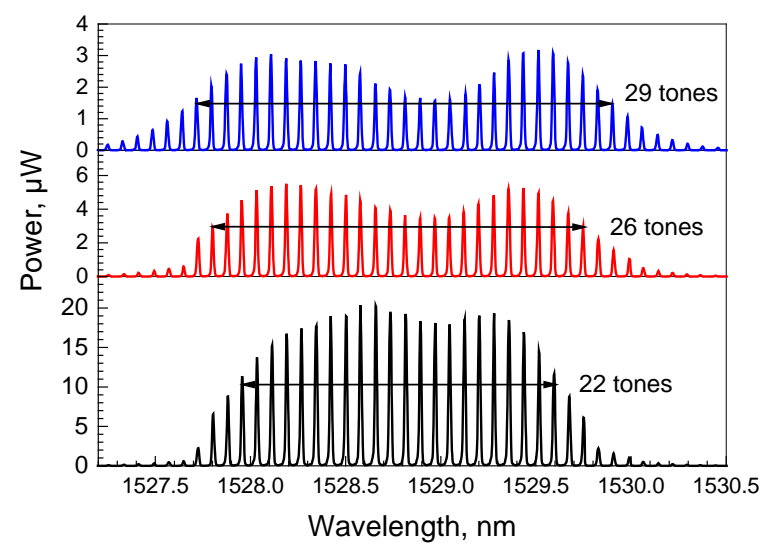

Fig. 3: Comb response when both RF signals were $26 / 27 \mathrm{dBm}$ for completely flat (22 tones) comb and comb with increased dip in the middle (26 and 29 tones).

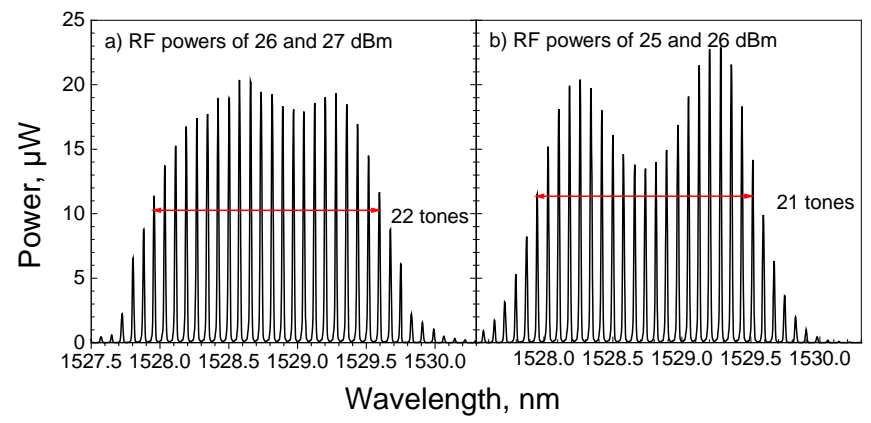

Fig. 4: Comb response when both RF signals were $26 / 27 \mathrm{dBm}$ (a) and 25/26 dBm (b).

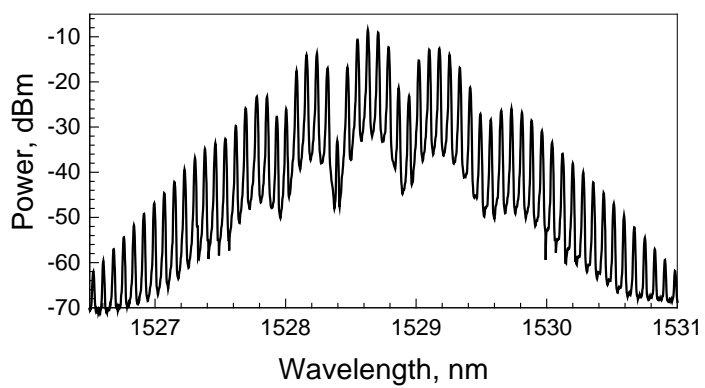

Fig. 5: The 'spectral beating' due to detuning of phase $\Delta \phi$ (about $\pi / 5$ from the optimally-flat condition) when its spectral period is about 65 GHz. 
In our experiments, we used a modulator with $10 \mathrm{GHz}$ electro-optic bandwidth. However, InP modulators can be designed to have higher bandwidths [17], allowing for the generation of combs with much wider spacing and thus also covering significantly wider spectral ranges. Using the approach suggested here for doubling the repetition rate, combs with large spacing, e.g. up to $100 \mathrm{GHz}$ would be possible, with RF drive electronics operating at half the comb spacing, implying better energetic efficiency and lower cost.
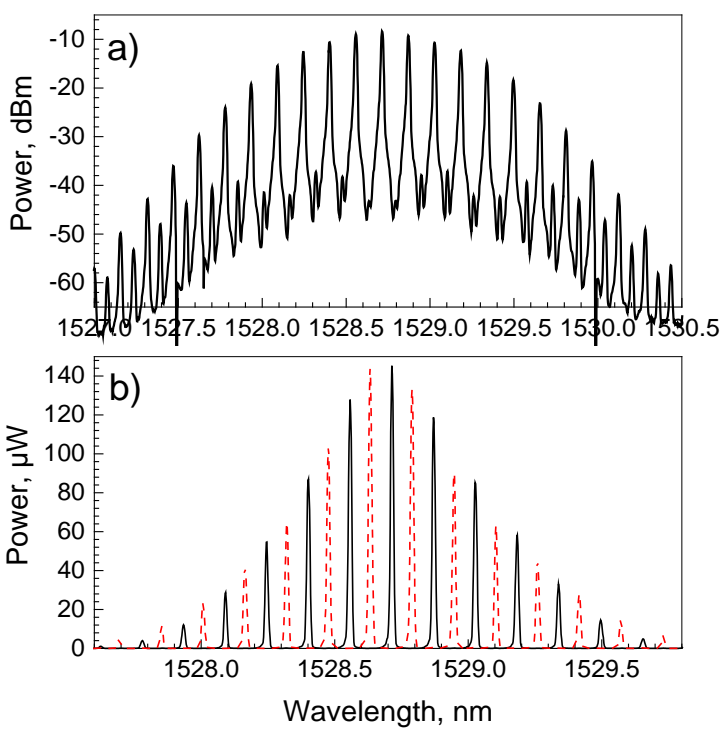

Fig. 6: Phase $\Delta \phi$ tuned by about $\pi$ from the optimally-flat condition, resulting in suppression of every other tone. (a) log scale when $\Delta \phi$ tuned to obtain only odd tones, showing the suppression of the even tones. (b) both cases - odd (dashed) and even (solid) tones suppressed in linear scale.

\section{CONCLUSIONS}

We investigated the suitability of an InP-based dual-electrode (push-pull) modulator to generate a flat optical comb. As compared to results published with $\mathrm{LiNbO}_{3}$ modulators, we obtained 50\% more comb tones (29) with almost $10 \mathrm{~dB}$ lower RF drive powers (26 and $27 \mathrm{dBm}$ ), whilst in comparison with results reported previously with an InP device, we observed improved flatness and a threefold increase in the number of comb tones generated. The modulator was driven close to the bandgap edge, resulting in a lower $\mathrm{V} \pi$ voltage, allowing for more efficient comb generation. The residual amplitude modulation associated with operating close to the band edge, unwanted in telecom applications, is demonstrated here to have no negative effects for comb generation. This is because the spectral asymmetry arising from this effect was set to have an opposite sign on the two RF electrodes, allowing for its efficient compensation at the modulator output. We also demonstrate generation of combs having twice the repetition rate of the comb spacing via control of the relative phase of the RF signals, allowing for the generation of combs beyond the electro-optic bandwidth of the device. The fundamental frequency suppression was better than $25 \mathrm{~dB}$. The device is simple (only one Mach-Zehnder modulator), resulting in low propagation loss and complexity, whilst offering performance (number of comb tones) only previously obtained using more complex schemes. The required RF power is modest, especially in comparison to that needed for $\mathrm{LiNbO}_{3}$ modulators. Being InP, the device has a very small footprint. It is also based on a device available commercially for a large market (Telecom), allowing it to represent a low-cost solution.

\section{REFERENCES}

[1] R.T. Watts, R. Rosales, C. Browning, E. Martin, K. Merghem, C. Calo, A. Martinez, A. Ramdane, and L.P. Barry, Characterization of optical frequency combs for OFDM based optical transmission systems, in International Conference of Fibre Optics and Photonics, Chennai, India, Dec 9-12, 2012, paper W2A.

[2] C.C. Renaud, M. Pantouvaki, S. Gregoire, I. Lealman, P. Cannard, S. Cole, R. Morre, R. Gwilliam, and A.J. Seeds, A monolithic MQW InPInGaAsP-based optical comb generator, IEEE J. of Quantum Electron., vol. 43, no. 11, pp. 998-1005, Nov 2007.

[3] P.M. Anandarajah, R. Maher, Y.Q. Xu, S. Latkowski, J. O’Carroll, S.G. Murdoch, R. Phelan, J. O’Gorman, and L.P. Barry, Generation of coherent multicarrier signals by gain switching of discrete mode lasers, IEEE Photonics Journal, vol. 3, no. 1, pp. 112-122, Feb. 2011.

[4] K.P. Ho and J.M. Kahn, Optical frequency comb generator using phase modulation in amplified circulating loop, IEEE Photon. Technol. Lett., vol. 5, pp. 721-725, 1993.

[5] T. Sakamoto, T. Kawanishi, and M. Izutsu, Optoelectronic oscillator using a LiNbO3 phase modulator for self-oscillating frequency comb generation, Opt. Lett., vol. 31, no. 6, pp. 811-813, March 2006.

[6] ] S. Bennett, B. Cai, E. Burr, O. Gough, and A.J. Seeds, 1.8-THz bandwidth, zero-frequency error, tuneable optical comb generator for DWDM applications, IEEE Photon. Technol. Lett., vol. 11, no. 5, pp. 551-553, May 1999.

[7] M. Kourogi, T. Enami, and M. Ohtsu, Monolithic optical frequency comb generator, IEEE Photon. Technol. Lett, vol 6, no 2, pp 214-217, 1994.

[8] T. Sakamoto, T. Kawanishi, and M. Izutsu, Asymptotic formalism for ultraflat optical frequency comb generation using a Mach-Zehnder modulator, Opt. Lett., vol. 32, no. 11, pp. 1515-1517, June 2007.

[9] T. Sakamoto, T. Kawanishi, and M. Izutsu, Widely wavelength-tunable ultra-flat frequency comb generation using conventional dual-drive Mach-Zehnder modulator, Electron. Lett., vol. 43, no. 19, May 2007.

[10] A.K. Mishra, R. Schmogrow, I. Tomkos, D. Hillerkuss, Ch. Koos, W. Freude, and J. Leuthold, Flexible RF-based comb generation, IEEE Photon. Technol. Lett., vol. 25, no. 7, pp. 701-704, April 2013.

[11] T. Yamamoto, K. Hitomi, W. Kobayashi, and H. Yasaka, Optical frequency comb block generation by using semiconductor MachZehnder modulator, IEEE Photon. Tech. Lett., vol. 25, no. 1, Jan 2013.

[12] T. Saikai, T. Yamamoto, H. Yasaka, and E. Yamada, Flat-top optical frequency comb block generation using InP-based Mach-Zehnder modulator, in $25^{\text {th }}$ International Conference on Indium Phosphide and Related Materials,, May 19-23, 2013, Kobe, Japan.

[13] C. Weimann, P.C. Schindler, R. Palmer, S. Wolf, D. Bekele, D. Korn, J. Pfeifle, S, Koeber, R. Schmogrow, L. Alloatti, D. Elder, H. Yu, W. Bogaerts, L.R. Dalton, W. Freude, J. Leuthold, and C. Koos, Siliconorganic hybrid (SOH) frequency comb sources for terabit/s data transmission, Opt. Express, vol. 22, no. 3, pp. 3629-3637, Feb. 2014.

[14] I.L. Gheorma and G.K. Gopalakrishnan, Flat frequency comb generation with an integrated dual-parallel modulator, IEEE Photon. Technol. Lett., vol. 19, no. 13, July 2007.

[15] Q. Wang, L. Huo, Y. Xing, and B. Zhou, Ultra-flat optical frequency comb generator using a single-driven dual-parallel Mach-Zehnder modulator, Opt. Lett., vol. 39, no. 10, pp. 3050-3053, May 2014.

[16] B. Betty, M. G. Boudreau, R. A. Griffin, and A. Feckes, An Empirical Model for High Yield Manufacturing of $10 \mathrm{~Gb} / \mathrm{s}$ Negative Chirp InP Mach-Zehnder Modulators," in Optical Fiber Communication Conference (OFC) paper OWE5 March 2005.

[17] R.A. Griffin, S.K. Jones, N. Whitbread, S.C. Heck, and L.N. Langley, InP Mach-Zehnder Modulator Platform for 10/40/100/200-Gb/s Operation, IEEE J. of Sel. Topic in Quant. Electron. Vol. 19, no. 6, article 3401209, Nov/Dec 2013. 